\title{
Depletion of the triggering receptor expressed on myeloid cells 2 inhibits progression of renal cell carcinoma via regulating related protein expression and PTEN-PI3K/Akt pathway
}

\author{
HAOJIE ZHANG ${ }^{1 *}$, LU SHENG $^{1 *}$, JING TAO $^{1}$, RAN CHEN $^{1}$, YANG LI $^{2}$, ZHONGQUAN SUN $^{1}$ and WEIQING QIAN ${ }^{1}$ \\ ${ }^{1}$ Department of Urology, Huadong Hospital, Fudan University, Shanghai; \\ ${ }^{2}$ Department of Biology, School of Life Science, Anhui Medical University, Hefei, Anhui, P.R. China
}

Received August 9, 2016; Accepted October 3, 2016

DOI: $10.3892 / \mathrm{ijo} .2016 .3740$

\begin{abstract}
The triggering receptor expressed on myeloid cells 2 (TREM-2) is suggested to be involved in the development of certain human malignancies. However, the functions of TREM-2 in renal cell carcinoma (RCC) are still less known. To reveal the effects of TREM-2 on the RCC progression, we examined the TREM-2 expression in RCC tumor tissues. Then, we analyzed the cell proliferation, cell apoptosis, cell cycle and expression of the relative factors in two selected RCC cell lines post RNA interference. We also analyzed the functions of TREM-2 in an in vivo nude mouse model. We found that, the expression of TREM-2 was abnormally elevated in RCC tumor tissues. Silencing TREM- 2 inhibited cell growth, induced G1 phase arrest of cell cycle and cell apoptosis in RCC cells. In vivo, the results showed that depletion of TREM-2 significantly inhibited the ACHN tumor growth in the nude mouse model. The analysis of relative protein factors suggested that silencing TREM-2 downregulated the expression levels of $\mathrm{Bcl} 2$ and PCNA, and upregulated the expression levels of Bax and caspase-3 in RCC cell lines. Depletion of TREM-2 inactivated PI3K/Akt pathway through increasing the expression of PTEN. Taken together, TREM-2 acts as an oncogene in the development of RCC and can be considered as a novel therapeutic factor in the treatment of RCC.
\end{abstract}

\section{Introduction}

Kidney cancer, one of the most common malignancies in genitourinary system, affects approximately 208,500 people all around the world each year. In addition, the global incidence of kidney cancer is continuously increasing (1). Renal cell

Correspondence to: Dr Weiqing Qian, Department of Urology, Huadong Hospital, Fudan University, West Yan'an Road 221, Shanghai 200040, P.R. China

E-mail: qwq222@vip.sina.com

${ }^{*}$ Contributed equally

Key words: TREM-2, renal cell carcinoma, cell proliferation, cell apoptosis, PTEN-PI3K/Akt pathway carcinoma (RCC) is identified as the most common type of kidney cancer, which is responsible for approximately $90-95 \%$ of primary kidney cancer cases (2). RCC starts in the cells of the proximal renal tubular epithelium, with classic symptoms including haematuria, flank pain and an abdominal mass. Although smoking, NSAIDs medication and family history are suggested as risk factors, the exact pathogenies of RCC remain poorly understood $(3,4)$. Besides, the therapy for RCC is still limited. Surgery is applied primarily in RCC treatment, as RCC is often insensitive to chemotherapy and radiotherapy. Whereas, surgery is not always efficient when the cancer has spread around the body. In recent years, target therapy has improved the treatment of RCC (5). Neutralization of vascular endothelial growth factor is proved to prolong the time to progression of disease in RCC patients. Thus, the research on potential targeting factors may light up the prospect for RCC treatment.

The transmembrane glycoproteins of triggering receptor expressed on myeloid cells (TREM) belong to the single immunoglobulin variable (IgV) domain receptor family (6). TREMs map to human chromosome 6p21.1 and encode TREM-1, TREM-2, TREM-4, TREM-5 and TREM-like genes in human. TREM-2 is encoded by a 1041-nucleotide long cDNA. This receptor consists of an extracellular domain, a transmembrane region and a short cytoplasmic tail $(7,8)$. TREM-2 is involved in many biological processes (9). In bone remodeling, TREM-2 has been suggested to favor osteoclast differentiation and morphology (10). In immune responses, TREM-2 is associated with the upregulation of CD40, CD86, MHC class II in dendritic cells and the maturation of dendritic cells $(11,12)$. TREM-2 also plays a role in suppressing the production of TNF and IL-6 and TLR signaling in macrophages $(13,14)$. Moreover, TREM-2 is also involved in the pathology of some diseases. Related research proved that the defects in TREM-2 may be a cause of polycystic lipomembranous osteodysplasia with sclerosing leukeoncephalopathy (PLOSL) (15), and a rare missense mutation (rs75932628-T) in TREM-2 may confer an obvious risk of Alzheimer's disease (16). Recently, TREM-2 is suggested functioning in human malignancies. Wang et al (17) have indicated that highly expressed TREM-2 promotes cell proliferation and invasion in glioma cells. Other studies also suggest that abnormally expressed TREM-2 may be associated 
with tumor immune evasion in lung cancer (18). Whereas, the effects of TREM-2 on RCC are still less known.

In the present study, we revealed the biological effects of TREM-2 on RCC cells for the first time. We found that the expression of TREM-2 is abnormally elevated in RCC tumor tissues. TREM-2 functioned as an oncogene in both RCC cell lines and tumor-bearing mouse model in vivo. The effect of TREM2 on RCC progression might be related to the regulation of apoptotic proteins and PTEN-PI3K/Akt pathway. Therefore, TREM-2 may provide a novel approach to the therapy for RCC.

\section{Materials and methods}

Tissue samples. Renal tumor tissues and adjacent normal tissues were collected from 40 patients with RCC treated at the Huadong Hospital, Fudan University. The tissues were stored at $-80^{\circ} \mathrm{C}$ until being used. The study was approved by the Ethics Committee of Huadong Hospital, Fudan University. Informed and written consent were obtained from all patients according to the guidelines of the ethics committee.

Cell culture. Five RCC cell lines, Caki-1, Caki-2, ACHN, 786-0 and OS-RC-2 were purchased from the American Type Culture Collection (ATCC; Manassas, VA, USA). Cells were cultured in RMPI-1640 medium supplemented with $10 \%$ fetal bovine serum (FBS; Gibco), $100 \mathrm{U} / \mathrm{ml}$ penicillin and $100 \mu \mathrm{g} / \mathrm{ml}$ streptomycin. Cell culture was maintained at $37^{\circ} \mathrm{C}$ in a humidified $5 \% \mathrm{CO}_{2}$ atmosphere.

RNA interference. To knock down the expression of TREM-2 in RCC cells, siRNA transfection was performed. siRNAs targeting three positions of human TREM-2 mRNA (NM_001271821.1; siRNA1: 125-147UCUUACUCUUUGUC ACAGA, siRNA1: 386-408 UUACGCUGCGGAAUCUACA and siRNA3: 591-613 GAGACACGUGAAGGAAGAU) were synthesized. A non-specific scramble siRNA sequence served as negative control (NC). siRNAs were transfected into RCC cells using Lipofectamine 2000 (Invitrogen) according to the manufacturer's instructions. The following assays were performed at $48 \mathrm{~h}$ post-RNA interference.

CCK-8 assay. To analyze the cell proliferation, Caki-2 and ACHN cells $\left(5 \times 10^{3}\right)$ were seeded into 96 -well plates and examined at $0,24,48$ and $72 \mathrm{~h}$ after siRNA transfection using commercial Cell Counting kit (7seabitech) per the instructions of the manufacturer. Absorbance excited at $450 \mathrm{~nm}$ of reacted cells were detected to valuate cell growth.

Cell cycle assay. At $48 \mathrm{~h}$ after siRNA transfection, Caki-2 and ACHN cells were collected and fixed by $70 \%$ ethanol at $-20^{\circ} \mathrm{C}$ for $2 \mathrm{~h}$. After being washed with phosphate-buffered saline (PBS), the cells were incubated with propidudium iodide (PI; $0.05 \mathrm{mg} / \mathrm{ml}$; Sigma-Aldrich) in the dark for $30 \mathrm{~min}$. In addition, cell cycle assay was performed using flow cytometer (BD Biosciences, San Jose, CA, USA) and analyzed using FlowJo cell cycle analysis software.

Cell apoptosis assay. Caki-2 and ACHN cells were harvested at $48 \mathrm{~h}$ post-RNA interference. Cell apoptosis assay was performed using Annexin $\mathrm{V}$ apoptosis detection kit APC (eBioscience, San Diego, CA, USA). The cells double stained with Annexin V-fluorescein isothiocyanate (FITC) and PI were then examined by flow cytometer (BD Biosciences). At least 10,000 cells were obtained for each experiment.

Reverse transcription and real-time PCR ( $q R T-P C R)$. Total RNA was extracted from RCC cells and tissue samples using TRIzol reagent (Invitrogen). Reverse transcription was performed via cDNA Synthesis kit (Fermentas, Waltham, MA, USA). qRT-PCR was processed using a standard SYBR-Green PCR kit (Fermentas). All the procedures were performed according to the manufacturer's instructions. The cycle conditions were $10 \mathrm{~min}$ at $95^{\circ} \mathrm{C}, 40$ cycles of $15 \mathrm{sec}$ at $95^{\circ} \mathrm{C}$ and $45 \mathrm{sec}$ at $60^{\circ} \mathrm{C}, 15 \mathrm{sec}$ at $95^{\circ} \mathrm{C}, 1 \mathrm{~min}$ at $60^{\circ} \mathrm{C}$ followed by $15 \mathrm{sec}$ at $95^{\circ} \mathrm{C}$ and $15 \mathrm{sec}$ at $60^{\circ} \mathrm{C}$. GAPDH served as internal control. The primer sequences were the following: TREM-2 NM_001271821.1): primer F, 5'-TGGCACTCTCACCA TTACG-3' and primer R, 5'-CCTCCCATCATCTTCCTTC AC-3'; Bax (NM_004324.3): primer F, 5'-AGCTGAGCGAGT GTCTCAAG-3' and primer R, 5'-TGTCCAGCCCATGAT GGTTC-3'; Bcl2 (NM_000633.2): primer F, 5'-AGACCGAA GTCCGCAGAACC-3' and primer R, 5'-GAGACCACACTGC CCTGTTG-3'; PCNA (NM_002592.2): primer F, 5'-GCCTG ACAAATGCTTGCTGAC-3' and primer R, 5'-TTGAGTGC CTCCAACACCTTC-3'; caspase-3 (NM_004346.3): primer F, 5'-AACTGGACGTGGCATTGAG-3' and primer R, 5'-ACA AAGCGACTGGATGAACC-3'; GAPDH (NM_001256799.1): primer F, 5'-CACCCACTCCTCCACCTTTG-3' and primer R, 5'-CCACCACCCTGTTGCTGTAG-3'.

Western blotting. Tissue samples were collected and put into homogenizer to grind into tissue homogenate. Treated and untreated RCC cells were harvested and washed twice with PBS. Then, tissue homogenate and cells were disrupted in a radio-immunoprecipitation assay lysis buffer. After protein normalization, tissue and cell samples were separated in SDS-PAGE and transferred to a nitrocellulose membrane. The blots were then incubated with appropriate primary and secondary antibodies following blocked with 5\% skim milk. Visualization was performed using the enhanced chemiluminescence (ECL; Millipore, Billerica, MA, USA). The antibody list was as follows: TREM-2 (1:800, Ab86491; Abcam, Cambridge, MA, USA), PCNA (1:1,000, \#13110; Cell Signaling Technology Danvers, MA, USA), Bax (1:300, Sc-493; Santa Cruz Biotechnology, Santa Cruz, CA, USA), Bcl2 (1:300, Sc-492; Santa Cruz Biotechnology), caspase-3 (1:500, Ab44976; Abcam), PTEN (1:1,000, \#9188; Cell Signaling Technology), PI3K (1:1,000, Ab189403; Abcam), p-PI3K (1:1,000, Ab182651; Abcam), Akt (1:1,000,\#9272; Cell Signaling Technology), p-Akt (1:1,000, \#9271, Cell Signaling Technology), GAPDH (1:1,500, \#5174; Cell Signaling Technology) and HRP-labeled secondary antibodies (1:1,000, A0208, A0181, A0216; Beyotime Institute of Biotechnolgy, Haimen, China).

Nude mouse xenograft model. Animal experiments were approved and performed per the guidelines of Animal Care and Use Committee of Huadong Hospital, Fudan University (Shanghai, China). Twelve BALB/c nude mice aged 4-weeks 


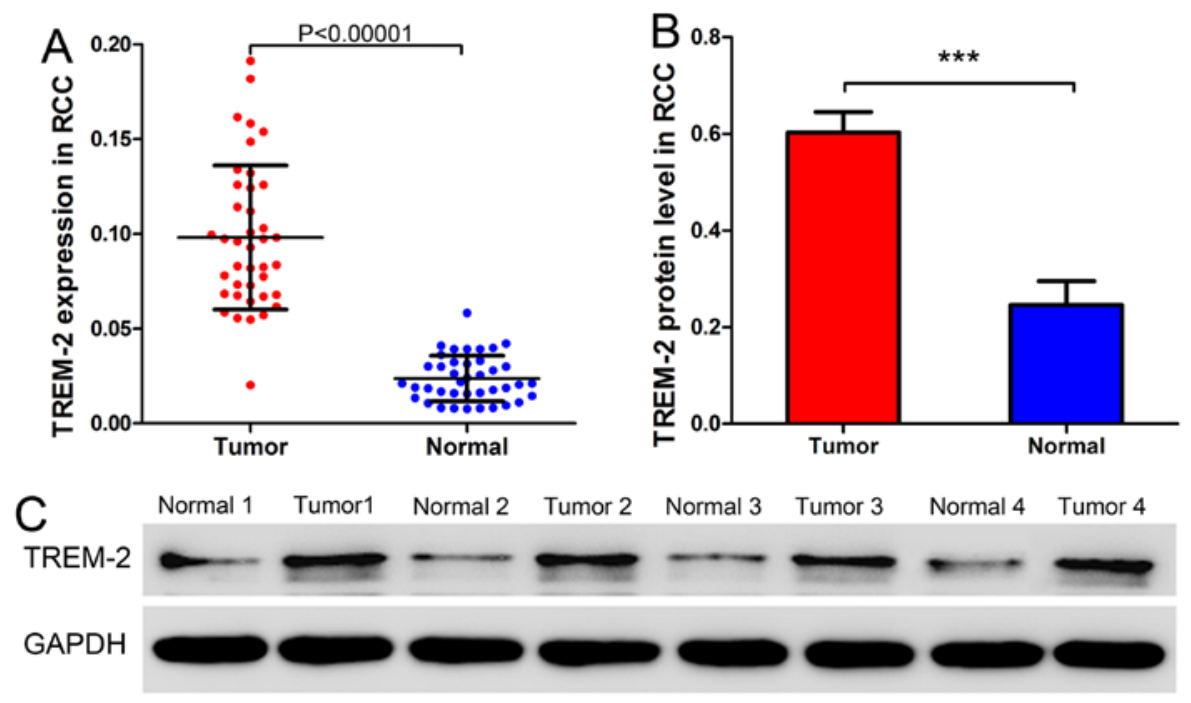

Figure 1. TREM-2 was elevated in RCC tissues. (A) The mRNA levels of TREM-2 in RCC tumor tissues were significantly higher than adjacent normal tissues $(n=40)$. (B and C) The protein level of TREM-2 was obviously increased in tumor tissues from patients with RCC ( $\mathrm{n}=40)$. Data are shown as mean \pm SD; **** $\mathrm{P}<0.001$.

(SLAC animal) were maintained under specific pathogen-free conditions using a laminar air-flow rack. All the mice were fed with sterilized food and autoclaved water. Injection was performed as previously described (19). Briefly, after one week of acclimatization, 12 nude mice were divided into two groups, NC group and siRNA group (n=6/group) randomly, and subcutaneously injected with ACHN cells transfected with NC-siRNA and TREM-2-siRNA $\left(2 \times 10^{6}\right)$ to the armpit of nude mouse, respectively. After 1 week of tumorigenesis, the shortest and longest diameter of the tumor were measured with calipers every 3 days, and tumor volume $\left(\mathrm{mm}^{3}\right)$ was calculated using the following standard formula: (the shortest diameter) ${ }^{2} \mathrm{x}$ (the longest diameter) $\mathrm{x} 0.5$. On day 34 post-injection, the mice were sacrificed by cervical dislocation and the tumors were harvested. The wet weights of each tumor were examined. During the experimental procedure, all mice were monitored every 2 days. None of the mice died prior to the experimental endpoint.

Statistical analysis. At least 3 independent experiments were performed in every assay. Data analysis used GraphPad Prism software. Data are expressed as mean $( \pm$ SD) and analyzed with t-test for multiple comparisons. $\mathrm{P}<0.05$ was considered to indicate a statistically significant result.

\section{Results}

TREM-2 expression is elevated in RCC tissues. To profile the expression pattern of TREM- 2 in RCC, we analyzed the mRNA and protein expression of TREM- 2 in tumor and adjacent normal tissues from 40 patients with RCC using qRT-PCR and western blot analysis. As Fig. 1 shows, both mRNA and protein levels of TREM2 were significantly higher in tumor tissues than adjacent normal tissues. These data indicated that the expression of TREM-2 was abnormally elevated in RCC tumor tissues.

TREM-2 knockdown via RNA interference. We then examined TREM-2 expression level in five RCC cell lines, including
Caki-1, Caki-2, ACHN, 786-0 and OS-RC-2, using qRT-PCR and western blot analysis. We found that TREM2 was highly expressed in ACHN and Caki-2 cell lines as comparing to other three cell lines (Fig. 2A and B). In addition, we employed these two RCC cell lines to carry out the following experiments. To further analyzed the biological functions of TREM-2 in RCC, TREM-2 expression was silenced in ACHN and Caki-2 cells using RNA interference. The results of qRT-PCR showed that, the mRNA level of TREM-2 was significantly suppressed after siRNA transfection. In addition, the siRNA2 (targeting position 386-408: UUACGCUGCGGAAUCUACA) silenced TREM-2 expression more effectively as comparing to the other two siRNAs. Thus, siRNA2 was selected as TREM2-siRNA transfected into cancer cells for the following assays. The western blot data also confirmed that siRNA2 decreased the protein level of TREM-2 in two RCC cell lines (Fig. 2C and D).

Depletion of TREM-2 inhibits cell proliferation in RCC cell lines. We analyzed the cell growth of ACHN and Caki-2 at 0 , 24, 48 and $72 \mathrm{~h}$ post-RNA interference using CCK-8 assay. As shown in Fig. 3, obvious decrease of cell growth was detected in both ACHN and Caki-2 cells at 48 and $72 \mathrm{~h}$ after siRNA transfection. Whereas, the cell proliferation was not affected in the NC group. These data suggested that knockdown of TREM-2 might suppress cell growth in RCC progression.

TREM-2 knockdown induces G1 phase arrest in RCC cell lines. As silencing TREM2 inhibited cell growth in RCC, we analyzed the effects of TREM-2 on the cell cycle progress in ACHN and Caki-2 cells sequentially. The RCC cells were stained by PI at $48 \mathrm{~h}$ post-RNA interference, and the cell cycle was examined by flow cytometry. As shown in Fig. 4, proportion of G1 phase was remarkably increased in siRNA-TREM-2 group than in the NC group, with increased ratios: 35.13 and $60.30 \%$ in ACHN and Caki-2, respectively. These results indicated that, silencing the expression of TREM-2 might inhibit cell cycle progress via inducing THE G1 phase arrest. 

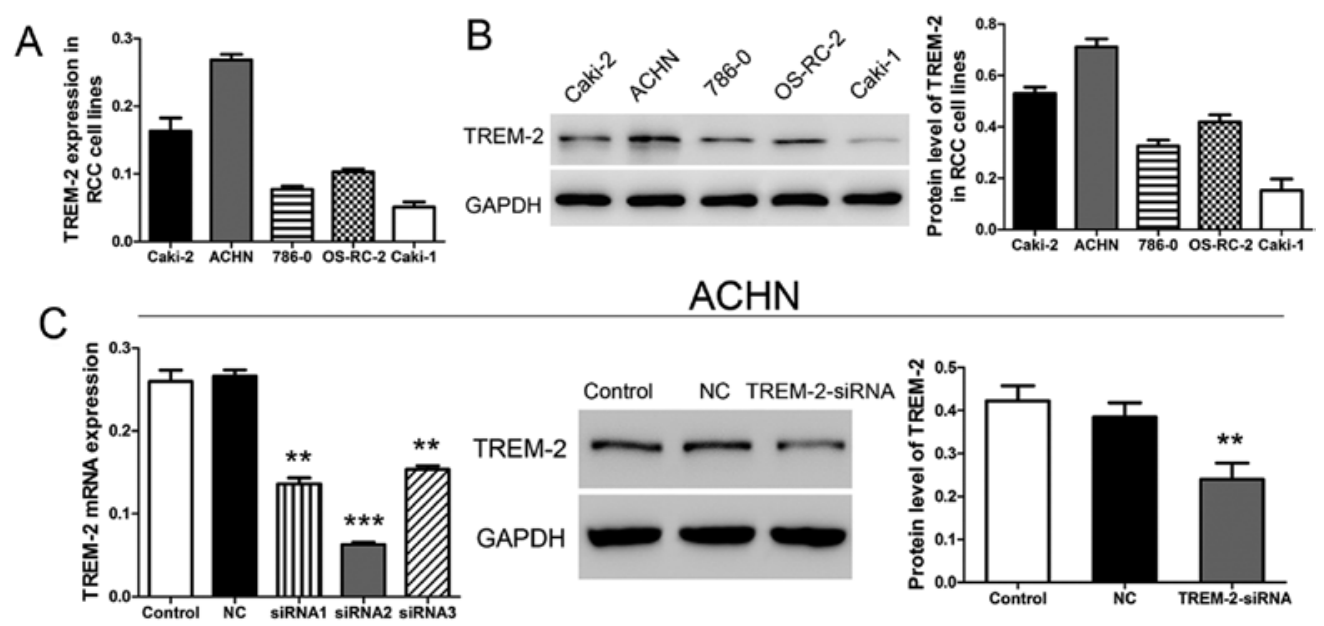

$\mathrm{ACHN}$

$\mathrm{D}$
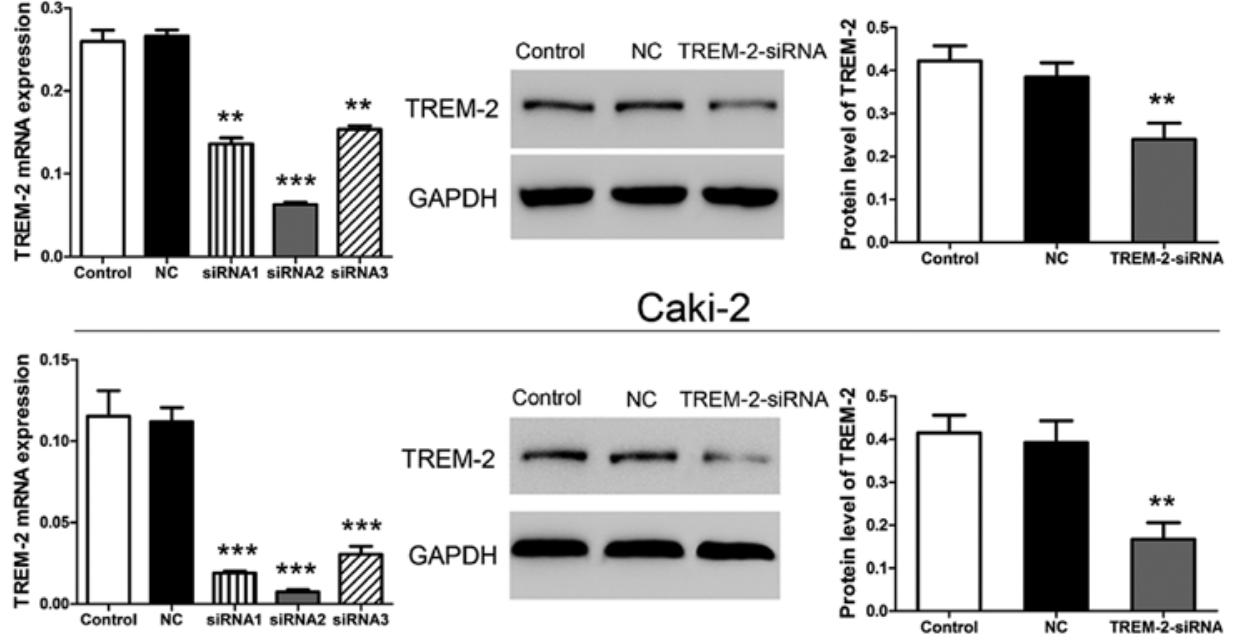

Caki-2

Figure 2. TREM-2 is suppressed in RCC cell lines post-RNA interference. (A and B) mRNA expression and protein level of TREM-2 in five RCC cell lines $(\mathrm{n}=3)$. ACHN and Caki-2 with highly expressed TREM-2 were selected for further assays. (C and D) ACHN and Caki-2 were transfected with three siRNAs $(\mathrm{n}=3)$. siRNA2 had more inhibitory effect on TREM-2 expression as comparing to other two siRNAs in both cell lines. In addition, siRNA2 was used as TREM-2-siRNA in subsequent experiments. The results of western blot assay confirmed that siRNA2 significantly decreased the intracellular protein level of TREM-2 ( $\mathrm{n}=3$ ). Data are shown as mean $\pm \mathrm{SD} ;{ }^{* * *} \mathrm{P}<0.01,{ }^{* * * *} \mathrm{P}<0.001$ (compared with negative controls).
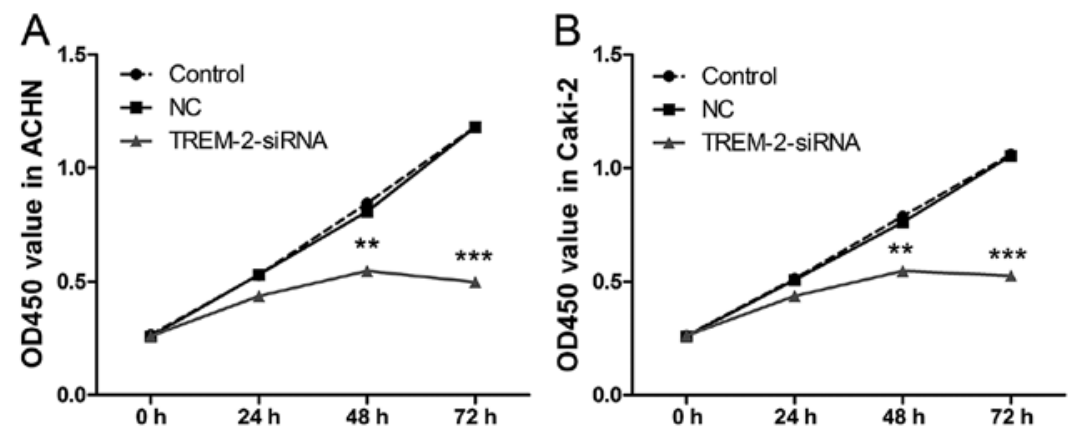

Figure 3. Silencing TREM-2 obviously inhibits cell proliferation in RCC cell lines. (A and B) Cell proliferation was analyzed using CCK-8 assay. The OD value was examined at 0, 24, 48 and $72 \mathrm{~h}$ post-RNA interference. The cell growth in ACHN and Caki-2 was significantly inhibited after TREM-2-siRNA transfection $(\mathrm{n}=3)$ as comparing to NCs. Data are shown as mean $\pm \mathrm{SD}$. ${ }^{* *} \mathrm{P}<0.01,{ }^{* * *} \mathrm{P}<0.001$ (compared with negative controls).

Silencing TREM-2 causes cell apoptosis in RCC cell lines. We analyzed the effects of TREM-2 on cell apoptosis in ACHN and Caki- 2 cells at $48 \mathrm{~h}$ after siRNA transfection. The cells were double stained by Annexin V-FITC and PI, and examined using flow cytometry. As illustrated in Fig. 5, 22-fold increase and 13-fold increase of cell apoptosis was examined in ACHN and Caki-2 cells of siRNA groups as comparing to NC groups. The data showed that knockdown of TREM-2 induced significant cell apoptosis in RCC cell lines.

Silencing TREM-2 inhibits xenografted ACHN tumor development in nude mice. We then examined whether TREM-2 silenced in RCC cells could inhibit tumor growth in vivo. The ACHN cells transfected with NC-siRNA or TREM-2-siRNa were subcutaneously injected into nude mice, respectively. Tumor volumes were measured for 27 days. The weight of tumors were measured on day 34. As shown in Fig. 6A-C, the mRNA expression and protein level of TREM-2 were significantly decreased in TREM-2-siRNA group compared with NC group. As shown in Fig. 6C-E, both the volume and the weight of RCC tumors were obviously decreased after TREM-2-siRNA transfection. These results implicated that TREM-2 knockdown might inhibit the tumor development of $\mathrm{RCC}$ in vivo.

Silencing TREM-2 altered endogenous expression of proteins related to apoptosis and the cell cycle in RCC cell lines. As described above, TREM-2 might act as a promoter in cell 


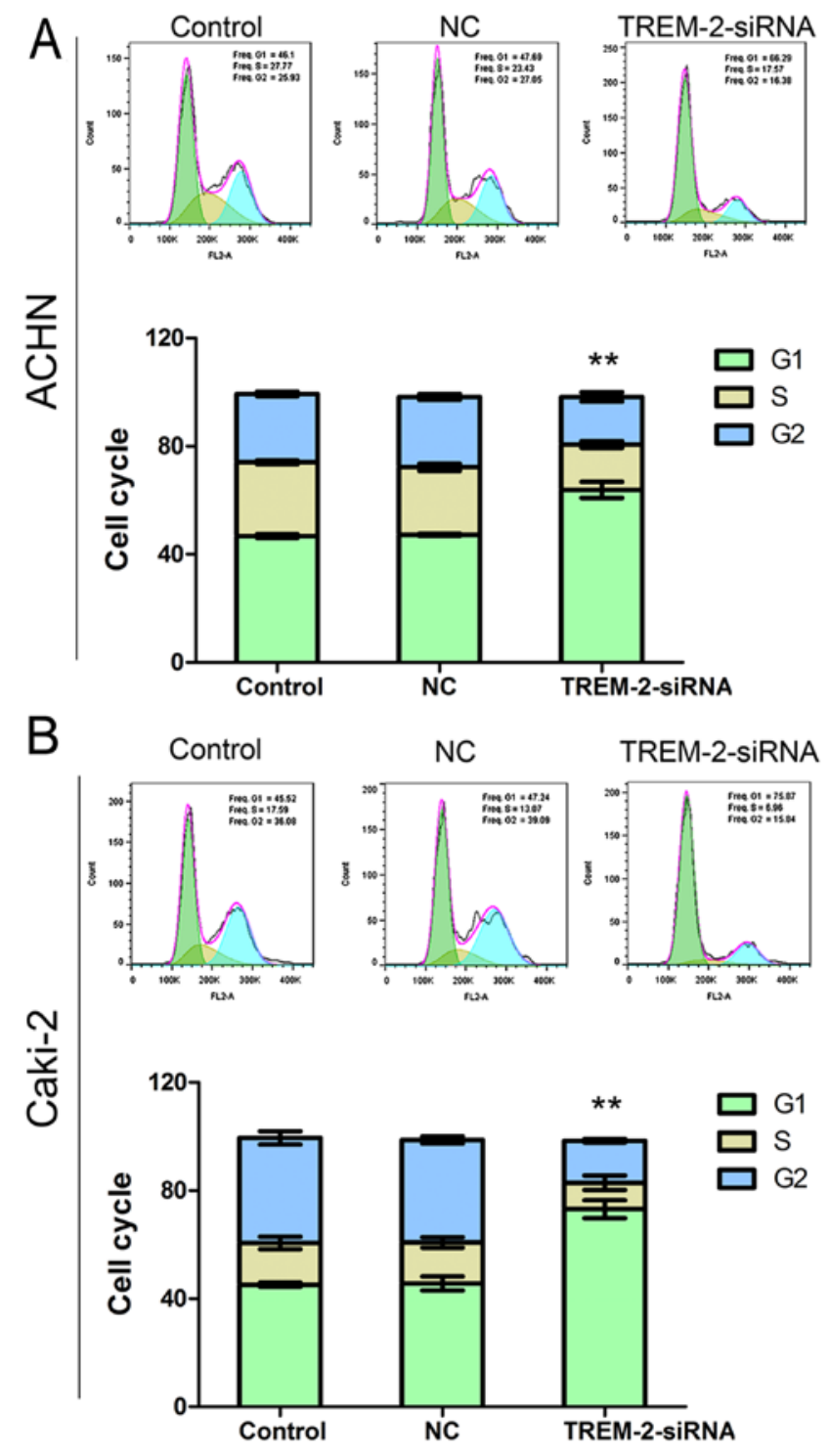

Figure 4. Silencing TREM-2 induces G1 phase arrest of cell cycle in RCC cell lines. (A and B) Transfected ACHN and Caki-2 cells were stained with PI and cell cycle profile was analyzed using flow cytometry $(n=3)$. The number of cells arrest in G1 phase was remarkably increased in TREM-2-siRNA groups. Data are shown as mean $\pm \mathrm{SD} ;{ }^{* *} \mathrm{P}<0.01$ (compared with negative controls).
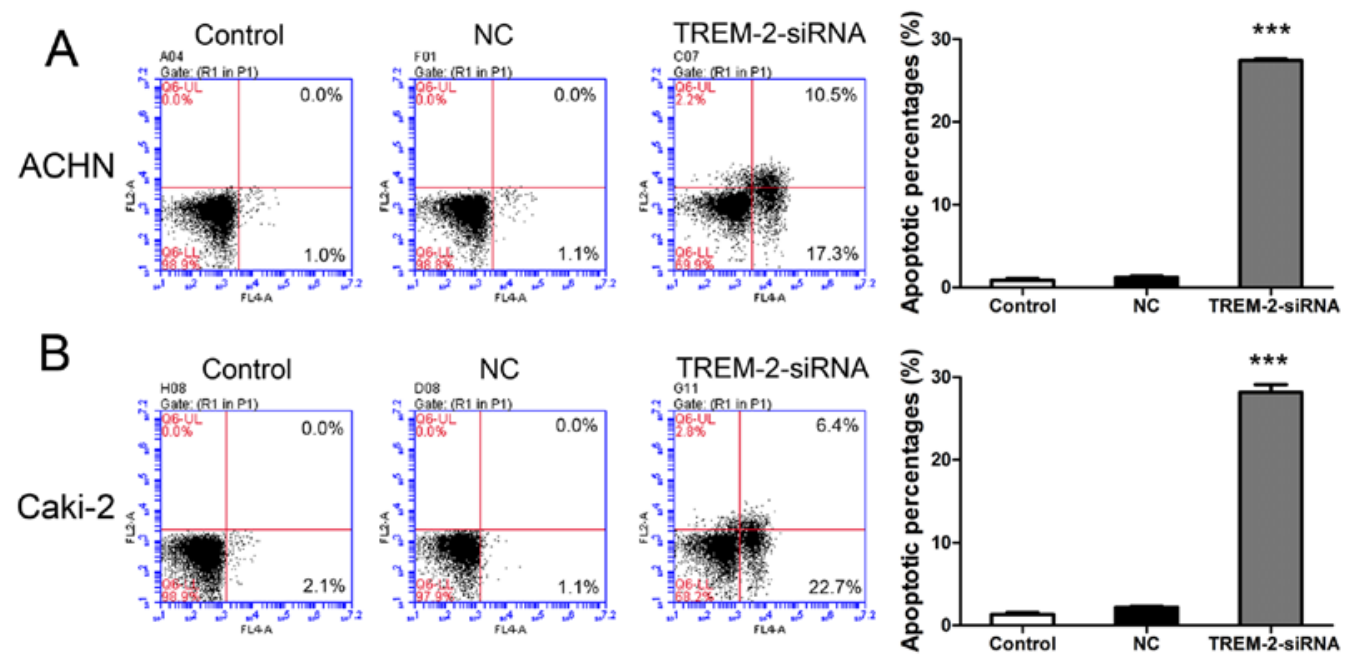

Figure 5. Silencing TREM-2 promotes cell apoptosis in RCC cell lines. (A and B) Transfected ACHN and Caki-2 were double stained with FITC and PI. Cell apoptosis was analyzed by flow cytometry $(n=3)$. The proportions of apoptotic cells at early or late phase were significantly increased after siRNA transfection. Depletion of TREM-2 obviously promoted cell apoptosis in both cell lines. Data are shown as mean $\pm \mathrm{SD} ;{ }^{* * * * *} \mathrm{P}<0.001$ (compared with negative controls). 

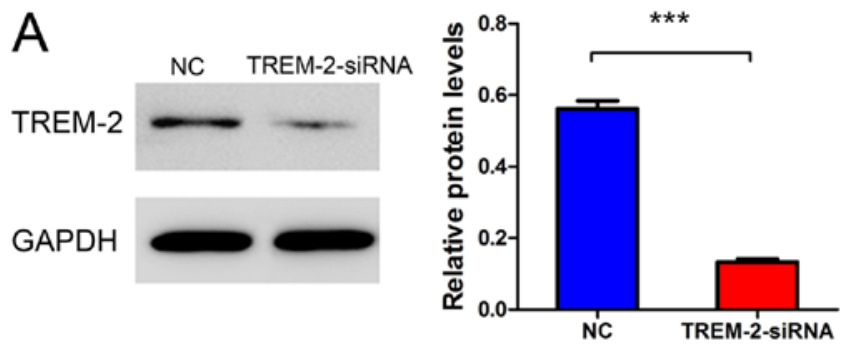

C NC TREM-2-siRNA

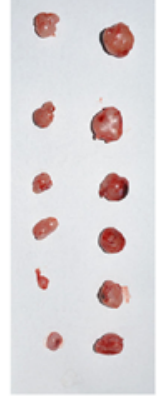

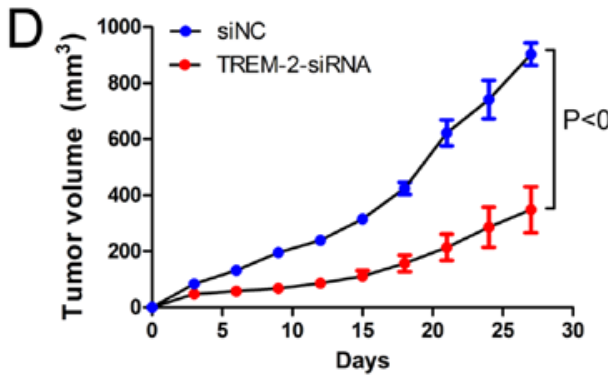
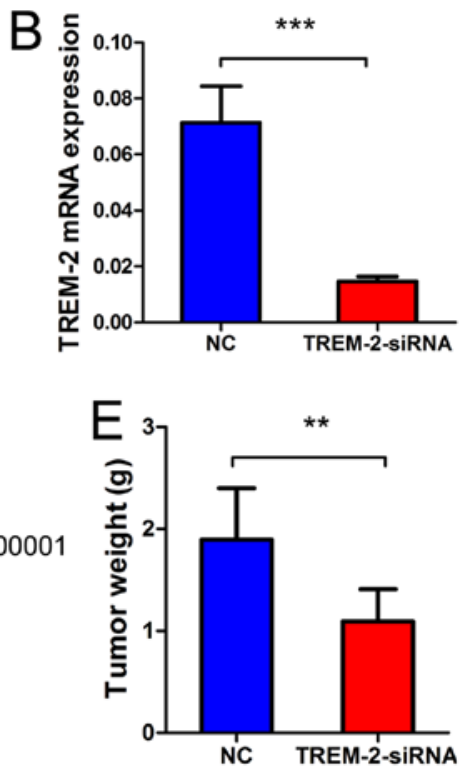

Figure 6. Silencing TREM-2 suppresses xenografted RCC tumor growth in nude mice. ACHN cells were transfected with TREM-2- and NC-siRNA, and expressed shRNA stably. The transfected cells were then subcutaneously injected into nude mice, respectively ( $\mathrm{n}=6$ ). (A and B) The mRNA expression at protein level of TREM-2 was obviously decreased in ACHN cells post-RNA interference. (C) Thirty-four days later, the mice were sacrificed by cervical dislocation and the tumors were harvested. Representative images of the ACHN tumors excised. (D and E) The tumor volumes and weights were measured at indicated time-points $(\mathrm{n}=6)$. These data indicated that knockdown of TREM-2 remarkably suppressed the ACHN tumor progression in vivo. Data are shown as mean $\pm \mathrm{SD} ;{ }^{* *} \mathrm{P}<0.01,{ }^{* * *} \mathrm{P}<0.001$ (compared with negative controls).

A

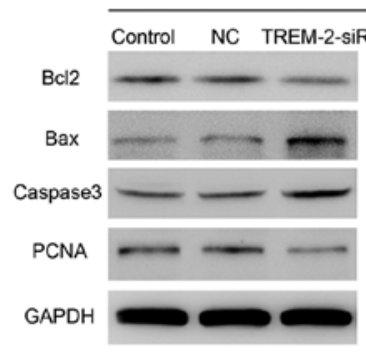

B
$\mathrm{ACHN}$

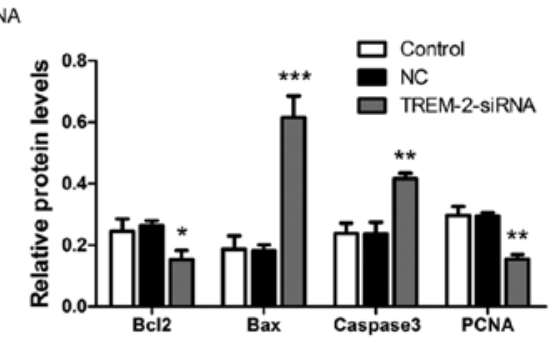

Caki-2

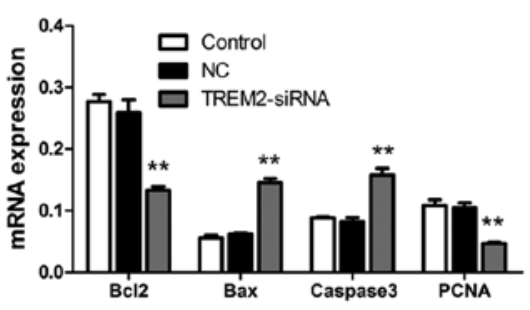

Caki-2

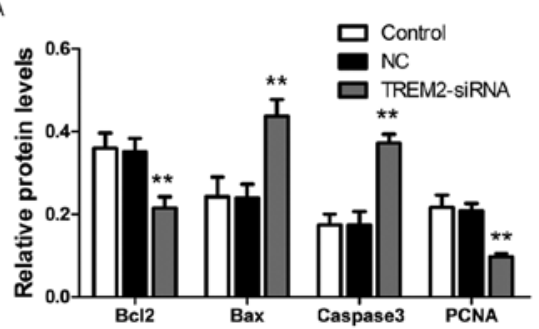

Figure 7. Silencing TREM-2 alters the expression of protein factors related to cell apoptosis in RCC cell lines. (A and B) The protein levels and mRNA expression of Bcl2, Bax, caspase-3 and PCNA in ACHN and Caki-2 were measured using western blot analysis and qRT-PCR (n=3). TREM-2 knockdown inhibited the expression of Bcl 2 and PCNA, but facilitated the expression of Bax and caspase-3. Data are shown as mean $\pm \mathrm{SD} ;{ }^{*} \mathrm{P}<0.05,{ }^{* *} \mathrm{P}<0.01,{ }^{* * *} \mathrm{P}<0.001(\mathrm{compared}$ with negative controls).

proliferation through inducing cell cycle progress and inhibiting cell apoptosis. We then examined four related protein levels, including Bcl2, Bax, caspase-3 and PCNA, using qRT-PCR and western blot analysis at $48 \mathrm{~h}$ post-RNA interference. As shown in Fig. 7, mRNA expression of Bcl2 (decreased ratios: $\mathrm{ACHN}, 48.55 \%$ and Caki-2, 80.47\%) and PCNA (decreased ratios: ACHN, 55.11\% and Caki-2, 69.58\%) were obviously decreased after TREM-2 knockdown. Whereas, the mRNA expression of Bax (increased ratios: ACHN, 134.16\% and Caki-2, 172.83\%) and caspase-3 (increase ratios: ACHN, $91.77 \%$ and Caki-2, 162.50\%) were significantly increased in siRNA-TREM-2 groups compared to NC groups. The same 
A

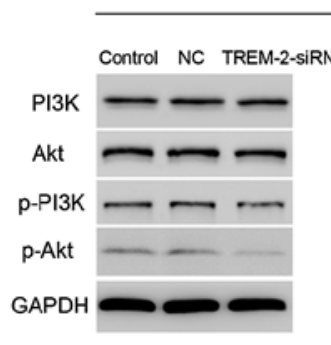

$\mathrm{ACHN}$
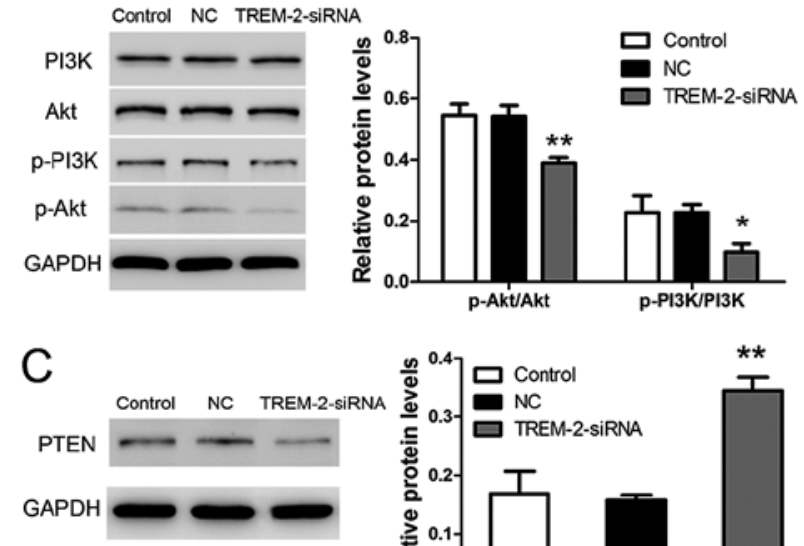

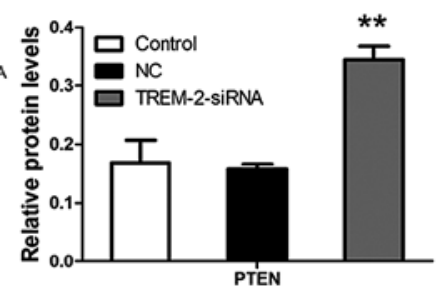

B
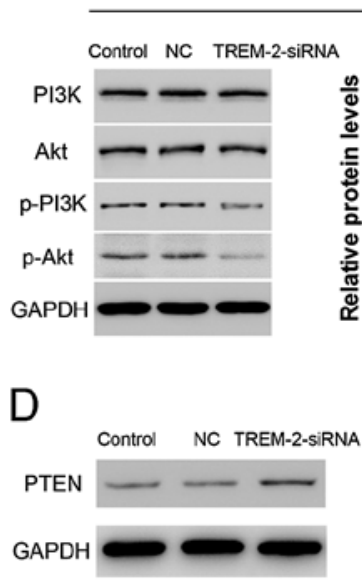

Caki-2
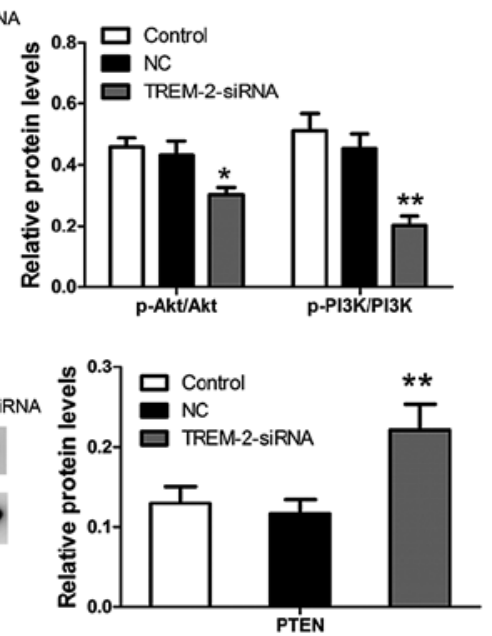

Figure 8. Silencing TREM-2 inactivates PI3K/Akt pathway via upregulating the level of PTEN in RCC cell lines. (A and B) Knockdown of TREM-2 significantly decreased the protein levels of p-PI3K and p-Akt in ACHN and Caki-2 cells. (C and D) Depletion of TREM-2 upregulated the intracellular level of PTEN in both cell lines. Data are shown as mean $\pm \mathrm{SD} ;{ }^{*} \mathrm{P}<0.05,{ }^{* *} \mathrm{P}<0.01$ (compared with negative controls).

results were also examined by western blot analysis. The protein levels of Bcl-2 and PCNA were reduced, while the protein levels of Bax and caspase- 3 were elevated after siRNA transfection. The decreased ratios of $\mathrm{Bcl} 2$ and PCNA protein levels were 41.94 and $47.39 \%$ in ACHN, 38.54 and $53.59 \%$ in Caki-2, respectively. The increased ratios of Bax and caspase-3 protein levels were 239.18 and $75.45 \%$ in $\mathrm{ACHN}$, and 82.99 and $113.98 \%$ in Caki-2, respectively.

Depletion of TREM-2 inactivates PTEN-PI3K/Akt signaling pathway in RCC cell lines. To reveal the functional mechanisms of TREM-2 in cell growth, we analyzed the PTEN-PI3K/ Akt signaling pathway using western blot analysis. As Fig. 8A and $B$ show, TREM-2 knockdown significantly increased the protein level of PTEN in ACHN and Caki-2 cells. Depletion of TREM-2 inhibited phoshoprylation of PI3K and Akt in both cell lines (Fig. 8C and D). These data suggested that TREM-2 knockdown inhibited the activation of PI3K/AKT pathway via upregulating the PTEN level in RCC cell lines.

\section{Discussion}

Immunotherapy and targeted therapy have recently provided new insights into the treatment of RCC. Research on novel targeting factors still remains urgent. TREM-1, a member of TREM family, has been suggested to be involved in progression of certain human malignancies. Liao et al (20) has proved that TREM-1 is related to the aggressive tumor behavior and has potential value as a prognostic factor for hepatocellular carcinoma. TREM-1 is upregulated in macrophages and is associated with cancer recurrence and poor survival of patients with lung cancer $(21,22)$. TREM-2 is also suggested to act as an oncogene in glioma (17). Moreover, TREM-2 is proven to promote tumor immune evasion in lung cancer cells (18). Inspired by the association between TREMs and human cancers, we sought to reveal the role of TREM-2 in RCC. We found that the expression of TREM-2 is remarkably facilitated in tumor tissues compared with the adjacent normal tissues of patients with RCC. It indicated that elevated TREM-2 might play a role in the RCC progression.

Then, we knocked down the TREM-2 expression in selected RCC cancer cell lines via RNA interference. In addition, we examined effects of TREM-2 depletion on cell proliferation, apoptosis and cell cycle in ACHN and Caki-2. The data showed that TREM-2 knockdown significantly inhibited cell growth, and induced cell apoptosis in two RCC cell lines. Similar results were also found in research by Wang et al (17). In their previous study, silencing TREM-2 suppressed cell proliferation and promotes cell apoptosis in glioma cells. In cell cycle assay, we found that depletion of TREM-2 induced arrest in G1 phase of cell cycle in RCC cells. In vivo, the data affirmed that knockdown of TREM-2 inhibited tumorigenesis of RCC cells. The above results illustrated that TREM-2 might function as an oncogene in RCC development.

To confirm the functional mechanisms of TREM-2 in RCC cells, we analyzed the protein levels and mRNA expression of factors related to apoptosis and cell cycle using western blot and qRT-PCR analysis. We found that in ACHN and Caki-2 cells, both mRNA expression and protein levels of Bcl2 and PCNA were obviously decreased post-RNA interference. Whereas, expression of Bax and caspase-3 were elevated in TREM-2siRNA groups as comparing to NC groups in two RCC cell lines. Bcl 2 belongs to the antiapoptotic Bcl2 family, which acts to prevent or delay cell death. Previous studies indicate that increased expression of Bcl2 in RCC may decrease the levels of apoptosis and promote resistant to treatment $(23,24)$. PCNA, a progressivity factor for DNA polymerase $\delta$, is essential for DNA replication $(25,26)$. In addition, suppression of PCNA may cause G1 phase arrest in cell cycle and promote cell death in cancer cells (27). Bax and caspase-3 act as promoters in cell apoptosis. Especially, activation of caspase-3 plays a central role in the execution-phase of cell apoptosis (28). Thus, the results of this study implicated that TREM-2 affects cell 
growth, apoptosis and cell cycle through regulating the mRNA expression and intracellular levels of related proteins.

Finally, we analyzed the effects of TREM-2 on PTENPI3K/Akt pathway. PI3K is a lipid kinase and generated $\mathrm{PI}(3,4,5) \mathrm{P} 3$, which is an essential second messenger in translocation of Akt to plasma membrane. Activated Akt plays a pivotal role in fundamental cellular functions $(29,30)$. The activation of PI3K/Akt pathway has been suggested involved in many kinds of human tumors, such as breast, lung cancer and leukemia (31-33). PI3K/Akt pathway also plays a key role in RCC. Activation of PI3K/Akt is associated with the decrease of the survival rate in RCC (34). Research has proven that certain anticancer agents, such as Klotho and $\beta$-elemene, inhibit the tumor progression through suppressing PI3K/Akt signaling in RCC (35-38). TREM-2 has been proven as an activator in PI3K/Akt signaling pathway. Elevated TREM-2 can mediate bacterial killing via activating PI3K/Akt pathway $(39,40)$. PTEN is identified as a tumor suppressor that is mutated in a large number of cancers at high frequency (41). PTEN acts as a key negative regulator in the alternations of PI3K/Akt through decreasing the intracellular level of $\mathrm{PI}(3,4,5) \mathrm{P} 3$ in cells (42). In addition, the disturbed expression and function of PTEN have been examined in both human cancer cell lines and human malignancies (43-45). Research has implicated that inhibition of PTEN may cause permanent activation of the PI3K/Akt pathway (46). In the present study, we found that TREM-2 knockdown significantly inactivated the PI3K/Akt pathway by downregulating the intracellular protein levels of p-PI3K and p-Akt, and increased the intracellular protein level of PTEN in two RCC cell lines. Therefore, the data suggested that TREM-2 might act as a regulator of PI3K/Akt through altering the PTEN level in RCC.

In conclusion, this study first revealed the role of TREM-2 in RCC progression. We found that TREM-2 is abnormally elevated in RCC tissues. Knockdown of TREM-2 obviously inhibited cell proliferation, induced cell apoptosis and G1 phase arrest in RCC cells. The in vivo experiments also confirmed that depletion of TREM-2 suppressed the tumor development of RCC. Moreover, the oncogene functional effects of TREM-2 might be caused by its regulation of related proteins and PTENPI3K/Akt pathway. Therefore, TREM-2 may be considered as a potential therapeutic target in the treatment of RCC.

\section{References}

1. Yi Z, Fu Y, Zhao S, Zhang X and Ma C: Differential expression of miRNA patterns in renal cell carcinoma and nontumorous tissues. J Cancer Res Clin Oncol 136: 855-862, 2010.

2. Rini BI, Campbell SC and Escudier B: Renal cell carcinoma. Lancet 373: 1119-1132, 2009.

3. Cheungpasitporn W, Thongprayoon $\mathrm{C}$, O'Corragain OA, Edmonds PJ, Ungprasert P, Kittanamongkolchai W and Erickson SB: The risk of kidney cancer in patients with kidney stones: A systematic review and meta-analysis. QJM 108: 205-212, 2014.

4. Ljungberg B, Campbell SC, Choi HY, Jacqmin D, Lee JE, Weikert S and Kiemeney LA: The epidemiology of renal cell carcinoma. Eur Urol 60: 615-621, 2011.

5. Singer EA, Gupta GN, Marchalik D and Srinivasan R: Evolving therapeutic targets in renal cell carcinoma. Curr Opin Oncol 25: 273-280, 2013

6. Allcock RJ, Barrow AD, Forbes S, Beck S and Trowsdale J: The human TREM gene cluster at $6 \mathrm{p} 21.1$ encodes both activating and inhibitory single IgV domain receptors and includes NKp44. Eur J Immunol 33: 567-577, 2003.
7. Klesney-Tait J, Turnbull IR and Colonna M: The TREM receptor family and signal integration. Nat Immunol 7: 1266-1273, 2006.

8. Whittaker GC, Orr SJ, Quigley L, Hughes L, Francischetti IM, Zhang W and McVicar DW: The linker for activation of B cells (LAB)/non-T cell activation linker (NTAL) regulates triggering receptor expressed on myeloid cells (TREM)-2 signaling and macrophage inflammatory responses independently of the linker for activation of T cells. J Biol Chem 285: 2976-2985, 2010.

9. Paradowska-Gorycka A and Jurkowska M: Structure, expression pattern and biological activity of molecular complex TREM-2/ DAP12. Hum Immunol 74: 730-737, 2013.

10. Filbert EA: Investigations of mechanisms involved in LPS-stimulated osteoclastogenesis. University of Connecticut, School of Dental Medicine. SoDM Masters Theses, Paper 155, 2007.

11. Sharif $O$ and Knapp S: From expression to signaling: Roles of TREM-1 and TREM-2 in innate immunity and bacterial infection. Immunobiology 213: 701-713, 2008.

12. Tomasello E, Desmoulins P-O, Chemin K, Guia S, Cremer H, Ortaldo J, Love P, Kaiserlian D and Vivier E: Combined natural killer cell and dendritic cell functional deficiency in KARAP/ DAP12 loss-of-function mutant mice. Immunity 13: 355-364, 2000.

13. Helming L, Tomasello E, Kyriakides TR, Martinez FO, Takai T, Gordon S and Vivier E: Essential role of DAP12 signaling in macrophage programming into a fusion-competent state. Sci Signal 1: ra11, 2008.

14. Turnbull IR, Gilfillan S, Cella M, Aoshi T, Miller M, Piccio L, Hernandez M and Colonna M: Cutting edge: TREM- 2 attenuates macrophage activation. J Immunol 177: 3520-3524, 2006.

15. Paloneva J, Manninen T, Christman G, Hovanes K, Mandelin J, Adolfsson R, Bianchin M, Bird T, Miranda R, Salmaggi A, et al: Mutations in two genes encoding different subunits of a receptor signaling complex result in an identical disease phenotype. Am J Hum Genet 71: 656-662, 2002.

16. Guerreiro R, Wojtas A, Bras J, Carrasquillo M, Rogaeva E, Majounie E, Cruchaga C, Sassi C, Kauwe JS, Younkin S, et al; Alzheimer Genetic Analysis Group: TREM2 variants in Alzheimer's disease. N Engl J Med 368: 117-127, 2013.

17. Wang XQ, Tao BB, Li B, Wang XH, Zhang WC, Wan L, Hua XM and Li ST: Overexpression of TREM2 enhances glioma cell proliferation and invasion: A therapeutic target in human glioma. Oncotarget 7: 2354-2366, 2016.

18. Yao Y, Li H, Wang Y and Zhou J: Triggering receptor expressed on myeloid cells-2 (TREM-2) elicited by lung cancer cells to facilitate tumor immune evasion. J Clin Oncol 31: (Suppl 22054, 2013.

19. Chen Y, Guo Y, Yang H, Shi G, Xu G, Shi J, Yin N and Chen D: TRIM66 overexpresssion contributes to osteosarcoma carcinogenesis and indicates poor survival outcome. Oncotarget 6: 23708-23719, 2015.

20. Liao R, Sun TW, Yi Y, Wu H, Li YW, Wang JX, Zhou J, Shi YH, Cheng YF, Qiu SJ, et al: Expression of TREM-1 in hepatic stellate cells and prognostic value in hepatitis B-related hepatocellular carcinoma. Cancer Sci 103: 984-992, 2012.

21. Ho CC, Liao WY, Wang CY, Lu YH, Huang HY, Chen HY, Chan WK, Chen HW and Yang PC: TREM-1 expression in tumor-associated macrophages and clinical outcome in lung cancer. Am J Respir Crit Care Med 177: 763-770, 2008.

22. Yuan Z, Mehta HJ, Mohammed K, Nasreen N, Roman R, Brantly $M$ and Sadikot RT: TREM-1 is induced in tumor associated macrophages by cyclo-oxygenase pathway in human non-small cell lung cancer. PLoS One 9: e94241, 2014.

23. Gobé G, Rubin M, Williams G, Sawczuk I and Buttyan R: Apoptosis and expression of Bcl-2, Bcl-XL, and Bax in renal cell carcinomas. Cancer Invest 20: 324-332, 2002

24. Sejima T and Miyagawa I: Expression of bcl-2, p53 oncoprotein, and proliferating cell nuclear antigen in renal cell carcinoma. Eur Urol 35: 242-248, 1999.

25. Fisher PA, Moutsiakis DL, McConnell M, Miller $\mathrm{H}$ and Mozzherin DJ: A single amino acid change (E85K) in human PCNA that leads, relative to wild type, to enhanced DNA synthesis by DNA polymerase $\delta$ past nucleotide base lesions (TLS) as well as on unmodified templates. Biochemistry 43: 15915-15921, 2004.

26. Zhang P, Sun Y, Hsu H, Zhang L, Zhang Y and Lee MY: The interdomain connector loop of human PCNA is involved in a direct interaction with human polymerase $\delta$. J Biol Chem 273: 713-719, 1998. 
27. Pleschke JM, Kleczkowska HE, Strohm M and Althaus FR: Poly(ADP-ribose) binds to specific domains in DNA damage checkpoint proteins. J Biol Chem 275: 40974-40980, 2000.

28. Cohen GM: Caspases: The executioners of apoptosis. Biochem J 326: 1-16, 1997.

29. Datta SR, Brunet A and Greenberg ME: Cellular survival: A play in three Akts. Genes Dev 13: 2905-2927, 1999.

30. Vivanco I and Sawyers CL: The phosphatidylinositol 3-Kinase AKT pathway in human cancer. Nat Rev Cancer 2: 489-501, 2002.

31. Fry MJ: Phosphoinositide 3-kinase signalling in breast cancer: How big a role might it play? Breast Cancer Res 3: 304-312, 2001.

32. Lin X, Böhle AS, Dohrmann P, Leuschner I, Schulz A, Kremer B and Fändrich F: Overexpression of phosphatidylinositol 3-kinase in human lung cancer. Langenbecks Arch Surg 386: 293-301, 2001.

33. Martínez-Lorenzo MJ, Anel A, Monleón I, Sierra JJ, Piñeiro A, Naval J and Alava MA: Tyrosine phosphorylation of the $\mathrm{p} 85$ subunit of phosphatidylinositol 3-kinase correlates with high proliferation rates in sublines derived from the Jurkat leukemia. Int J Biochem Cell Biol 32: 435-445, 2000.

34. Merseburger AS, Hennenlotter J, Kuehs U, Simon P, Kruck S, Koch E, Stenzl A and Kuczyk MA: Activation of PI3K is associated with reduced survival in renal cell carcinoma. Urol Int 80: 372-377, 2008

35. Zhu Y, Xu L, Zhang J, Xu W, Liu Y, Yin H, Lv T, An H, Liu L, $\mathrm{He} \mathrm{H}$, et al: Klotho suppresses tumor progression via inhibiting PI3K/Akt/GSK3 $\beta /$ Snail signaling in renal cell carcinoma. Cancer Sci 104: 663-671, 2013.

36. Zhan YH, Liu J, Qu XJ, Hou KZ, Wang KF, Liu YP and Wu B $\beta$-Elemene induces apoptosis in human renal-cell carcinoma 786-0 cells through inhibition of MAPK/ERK and PI3K/ Akt/ mTOR signalling pathways. Asian Pac J Cancer Prev 13: 2739-2744, 2012.
37. Li H, Zeng $\mathrm{J}$ and Shen K: PI3K/AKT/mTOR signaling pathway as a therapeutic target for ovarian cancer. Arch Gynecol Obstet 290: 1067-1078, 2014.

38. Roy SK, Srivastava RK and Shankar S: Inhibition of PI3K/AKT and MAPK/ERK pathways causes activation of FOXO transcription factor, leading to cell cycle arrest and apoptosis in pancreatic cancer. J Mol Signal 5: 10, 2010.

39. Sun M, Zhu M, Chen K, Nie X, Deng Q, Hazlett LD, Wu Y, Li M, Wu M and Huang X: TREM-2 promotes host resistance against Pseudomonas aeruginosa infection by suppressing corneal inflammation via a PI3K/Akt signaling pathway. Invest Ophthalmol Vis Sci 54: 3451-3462, 2013.

40. Zhu M, Li D, Wu Y, Huang X and Wu M: TREM-2 promotes macrophage-mediated eradication of Pseudomonas aeruginosa via a PI3K/Akt pathway. Scand J Immunol 79: 187-196, 2014.

41. Myers MP and Tonks NK: PTEN: Sometimes taking it off can be better than putting it on. Am J Hum Genet 61: 1234-1238, 1997.

42. Maehama T and Dixon JE: The tumor suppressor, PTEN/ MMAC1, dephosphorylates the lipid second messenger, phosphatidylinositol 3,4,5-trisphosphate. J Biol Chem 273: 13375-13378, 1998.

43. Eng C: PTEN: One gene, many syndromes. Hum Mutat 22: 183-198, 2003.

44. Muñoz J, Lázcoz P, Inda MM, Nistal M, Pestaña A, Encío IJ and Castresana JS: Homozygous deletion and expression of PTEN and DMBT1 in human primary neuroblastoma and cell lines. Int J Cancer 109: 673-679, 2004.

45. Nassif NT, Lobo GP, Wu X, Henderson CJ, Morrison CD, Eng C, Jalaludin B and Segelov E: PTEN mutations are common in sporadic microsatellite stable colorectal cancer. Oncogene 23: 617-628, 2004.

46. Osaki M, Oshimura M and Ito H: PI3K-Akt pathway: Its functions and alterations in human cancer. Apoptosis 9: 667-676, 2004. 